\title{
Interrelationship of Nutrition and Immunity 1
}

\section{G. D. Butcher, DVM, Ph.D., R. D. Miles, Ph.D.²}

Poultry encounter numerous stressors during their lives. These stressors cause hormone changes, declines in feed intake, altered nutrient metabolism and suppressed immune function. The adverse effects of the stressors are additive and every attempt should be made in all poultry operations to lessen the number and intensity of these stressors. Nutrients are known to influence the responses of poultry to a disease challenge. Normally, during such a challenge nutrients are shunted away from growth. For example, body proteins are broken down and amino acids are shunted away from growth and are used by specific cells to synthesize critical proteins which allow the bird to mount a successful immune response to a particular disease challenge. Lymphoid cells increase in their numbers, and acute phase proteins and antibodies also increase through the use of amino acids. Defense mechanisms take priority in a disease challenge and these nutrients help the bird's system overcome the challenge. If specific nutrients are at or below the bird's requirement, then a limited amount of nutrients will be available to meet all of the body's needs during a time of challenge.

The immune system benefits greatly from proper nutrition of the bird. Not only does the immune system benefit directly from proper nutrition, but indirectly proper nutrition will also prepare the bird for periods of stress, reducing the adverse effects of stress and enhancing recovery from stressful periods. Therefore, in many instances, proper nutrition lessens the immune suppression associated with the stress response in the bird. The immune system of the bird can be influenced by nutrition in several ways:

1. Anatomical development of lymphoid tissues.

2. Mucus production.

3. Synthesis of immunologically active substances.

4. Cellular proliferation.

5. Cellular activation and movement.

6. Intracellular killing of pathogens.

7. Modulation and regulation of the immune process.

When an immune response occurs from challenge by a pathogen, a systemic acute phase or inflammatory response results that is considered to be the foundation of non-specific immunity. Antibody titers that arise as a result of the humoral

1. This document is VM139, one of a series of the Veterinary Medicine-Large Animal Clinical Sciences Department, Florida Cooperative Extension Service, Institute of Food and Agricultural Sciences, University of Florida. Original publication date June 12, 2002. Visit the EDIS Web Site at http://edis.ifas.ufl.edu.

2. Gary D. Butcher, DVM, Ph.D., Diplomate, American College of Poultry Veterinarians, University of Florida College of Veterinary Medicine, Gainesville, FL., Richard D. Miles, Ph.D., Professor, Poultry Nutritionist, Department of Dairy and Poultry Sciences. 
response are not a direct burden on the anima'ls pool of nutrients and are therefore nutritionally inconsequential. It is therefore the systemic acute phase response that has nutritional consequences for the animal. It is this response that is associated with a shift in nutrients use away from skeletal muscle accretion and towards hepatic secretion of acute phase proteins as well as cellular and macromolecule turnover (Klasing, 1998).

There is no question that a well-nourished bird is more immunologically competent and better able to cope with disease challenges than a poorly nourished bird. A change in feed intake leads to a change in nutrient and energy intake. The development and maturation of the immune system of young poultry fed nutritionally deficient diets will be compromised. However, young poultry in today's commercial poultry industry are usually not subjected to diets which are severely limited in nutrients and energy. It is well known that immunological challenge in the bird is accompanied by a decrease in feed intake. Many nutritionists increase the energy density of the diet when feed intake declines. Using fat as the energy source of choice in such instances may not be the best decision. Carbohydrate calories may be the best choice since immunological stress is known to impair triglyceride clearance from the blood, thus decreasing fat use. Literature data clearly show that for most nutrients, dietary concentrations, which are necessary for optimal growth, are also necessary for optimal immunocompetence. However, data collected in studies with poultry and other species have shown that when a diet is deficient in protein or amino acids, the concentration of circulating antibodies to a specific challenge organism is low.

Recently, Kidd et al., (2001) reported that a dietary arginine concentration near the $1.25 \%$ of the diet recommended by the NRC (1994) should support proper immune system functions in healthy chicks. Also, these researchers reported that dietary arginine and lysine fed near the NRC (1994) requirement did not interact to affect immunity of chicks and cellular and humoral immune system functions of healthy chicks were not improved by fortified diets with aginine levels in excess of the NRC (1994) recommendation. This essential amino acid, arginine, plays a very important role as a potent immunological modulator since the cellular metabolic pathway of arginine produces nitric oxide (Collier and Vallance, 1989). Sung et al., (1991) found that nitric oxide production of macrophages is increased by a local concentration of arginine. Additional improvements in immunity, as affected by dietary arginine in animals including humans, include improved thymic weight and function, enhanced lymphocyte mitogenesis, improved immunity against tumors and enhanced wound healing (Efron and Barbul, 1998; Evoy et al., 1998).

A reduced humoral immune response in poultry has been associated with low mineral levels in the feed. For example, sodium and chloride have been associated with the humoral immune response in broilers. Broilers fed diets deficient in sodium or chloride have lower antibody concentrations in their bodies. The balance between sodium and chloride in the diet is important because a high chloride concentration in relation to that of sodium may result in a reduced immune response.

Even though most nutrients required by the immune system are present in the diet in sufficient concentrations there is evidence that increased dietary supplementation of certain nutrients, above that needed for maximum growth and feed efficiency, is of benefit to the immune response. Vitamins A, E and $\mathrm{C}$ as well as the amino acids methionine and valine have been shown to benefit immune function when added to the diet of poultry at higher concentrations than are required to maximize growth and feed efficiency. However, too much of a good thing may also be detrimental to the immune system. For instance, too much dietary methionine will suppress the immune system. Usually, the immune suppression caused by excess dietary methionine is evident before changes in growth and feed consumption are detected. It is essential to ensure that poultry diets contain enough of a specific nutrient to ensure the desired growth, feed conversion and immune response, but not too much so as to impair the immune function either directly or indirectly. The omega 3 and 6 fatty acids are also of benefit to the immune system at higher than normal concentrations in the diet. Several nutrients have been shown to have a negative effect on the immunological response in the bird when they are deficient in the diet. 
Sodium, chloride, zinc, methionine, valine, threonine, vitamin A, riboflavin, pantothenic acid, pyridoxine and selenium will have a negative impact on the immune system if they are deficient in the diet. Thus, these are considered essential to proper immune function.

One vitamin that has received a lot of attention with respect to its importance to the immune response in poultry has been vitamin $\mathrm{E}$. This vitamin appears to be an immune system "booster." Vitamin E seems to exert a complementary effect on the immune system by inhibiting the synthesis of prostaglandins. These prostaglandins are produced in the cells following the oxidation of cellular membranes and are responsible for inhibiting the inflammation and immune response. Vitamin E prevents oxidation and thus, the production of prostaglandins. It is well known that deficiencies of vitamin $\mathrm{E}$ and selenium will inhibit the immune response in poultry. Selenium works with vitamin $\mathrm{E}$ in tissues to protect biological membranes from oxidative damage. Zinc is another element that is important in assisting the bird's immune system to overcome a challenge. Zinc is especially important in wound healing, thymic function and proliferation of lymphocytes.

Leshchinsky and Klasing (2001) showed that supplementing broiler diets with vitamin $\mathrm{E}$ at 25 to 50 $\mathrm{IU} / \mathrm{Kg}$ of diet was not immunomodulatory and higher levels of vitamin E supplementation to the diet were less effective. As discussed by Gershwin et al., (1985) it is very likely that vitamin E, like other nutritional factors, affects the development and maintenance of immunocompetence through multiple factors, by acting directly on the immune cell or by indirectly altering metabolic and endocrine parameters, which in turn influence immune function. The main mechanism of the bioactivity of vitamin $\mathrm{E}$ is associated with its antioxidant potential in reducing free radical-induced pathology during normal metabolic stress and immune challenge. By controlling the production of free radicals, vitamin $\mathrm{E}$ affects free radical-mediated signal transduction events and ultimately modulates gene expression caused by free radical signaling (Packer and Suzuki, 1993). Vitamin $\mathrm{E}$ is also very important in preventing fatty acid peroxidation (Benedich, 1990). Fatty acids can act as immunoregulatory molecules that mediate cellular communication, membrane fluidity and second messenger elaboration (Klasing, 1997; Watkins, 1991). Another potential immunoregulatory mechanism that is associated with vitamin $\mathrm{E}$ is the modulation of arachidonic metabolism via cyclooxygenase and lipoxygenase pathways (Blumberg, 1994) which lead to the synthesis of prostaglandins and leukotrienes, respectively.

The effects of vitamin $\mathrm{E}$ on performance and immune response have been studied in our laboratory using commercial egg-type pullets fed diets supplemented with 10,50 and $100 \mathrm{IU} / \mathrm{kg}$ of vitamin E from one day through seven weeks of age and levels of 5, 25 and $50 \mathrm{IU} / \mathrm{kg}$ from eight through nineteen weeks of age, respectively. Tracheal lesion severity scores were evaluated four days post-injection challenge with a Massachusetts Infectious Bronchitis live field virus. Tracheal lesion scores decreased in a dose-related manner as dietary vitamin $\mathrm{E}$ levels increased. Thus, these data demonstrated the effectiveness of vitamin $\mathrm{E}$ supplementation in limiting the severity of disease after a challenge.

Disease challenge is only one of the many factors that will have an effect on the nutrient requirements of poultry. Insufficient nutrient consumption will reduce the effectiveness of the bird's defense mechanisms. Nutritionists must supply enough dietary nutrients and energy to allow the bird to express the desired growth and feed efficiency. Since the possibility of disease challenge is always present in today's poultry operations, the nutritionist must realize that the bird's metabolism and immune system are constantly adjusting to the stress of environment or disease and nutrient requirements may need to be increased at certain times.

It must not be forgotten that even though immune stimulation is essential for any animal to cope with the continual challenge of antigens, it is not without a cost. For instance, in broilers, immune stimulation is of extreme importance, but continued genetic selection for growth in broilers results in birds which have inefficient immune systems. Immune stimulation is associated with a catabolic response that results in a growth depression. This fact will continually cause a conflict between geneticists and 
nutritionists. Geneticists are interested in breeding faster growing birds. Accelerated growth is accompanied by impaired immune systems. Nutritionists attempt to promote immune stimulation realizing that maximal growth may not be attained if birds get sick. As a result it is not surprising that today commercial meat birds express their extremely high growth potential but seem to be more vulnerable to disease. Keeping this in mind, it must be realized that the acute phase response associated with immune, neurogenic or non-specific injury will boost the innate immune response rapidly and is associated with catabolism (Berczi et al., 1998).

Keeping flocks of poultry in excellent immunological health, so that they suffer no immuno-depression from any cause, especially nutrition, should be the goal of the company's veterinarian and nutritionist. Veterinarians and nutritionists working together with the production manager of the flocks make a very strong team which will be one of any poultry company's greatest assets.

\section{Stress: Its Effect on Nutrition and Immunity}

Anyone wishing to understand the interrelation between nutrition and immunity has to also understand that the stress response in the bird has an important influence on the above interrelationship. A three-way interrelationship exists among stress, nutrition and immunity and it is because of this that this paper emphasizes all three of these important factors that influence overall poultry performance.

The word stress means different things to different people and because of this, stress has been difficult to define. Stress is brought about by stress-producing factors, called stressors. It is important to be able to distinguish between a stressor and stress. Stress is the nonspecific response that the body of an animal has to any demand made upon it. An animal is under stress when it has to make extreme functional, structural, behavioral, or immunological adjustments to cope with adverse aspects of its environment (Curtis, 1983). A natural environment is composed of various potentially hostile stressors. Animals that are able to cope with the stressors to which they are exposed are those that will perform best in stressful situations. In nature, stress is the rule not the exception--after all, complete freedom from stress is death (Selye, 1973). Survival depends on the severity, duration, and interaction of the environmental stressors and the animal's physiological and behavioral ability to respond and adapt to them (Harvey et al., 1984). Once an animal perceives a stressor, its immediate response is usually behavioral (Friend an Dellmeier, 1986). It will tend to move away from the unpleasant stimulus. Depending on the severity and nature of the stressor, the autonomic nervous system responds, followed by a neuroendocrine response. These biological responses can eliminate or reduce the potential effects of the stressor by changing either the animal's relationship to the stressor or its perception of the stressor. If these responses are not successful, a pathological state may be induced that can result in decreased performance or a disease state.

Many of the problems in the poultry industry today are a direct result of the bird having to cope with one or a combination of stressors in the environment. Poor management is one of the greatest causes of stress in all types of poultry. The exposure to disease agents, poor nutrition and exposure to immunosuppressive agents also contribute to reduced performance. Poultry must be managed correctly in order to minimize the effects of stress on their performance and health. Successful identification and correction of problems in the poultry house and in the diet will benefit the bird. This paper will review some of the nutritional factors related to stress in poultry.

\section{Energy Need During Physiological Stress}

Whenever poultry are confronted with physiological stress they have to adapt to the situation in order to survive. This process of adaptation is essential and requires energy. The energy for adaptation comes from the three energy-yielding nutrients: carbohydrates, lipids and proteins. These nutrients are only available from the feed and the nutrient reserves in the animal. During the first stages of stress, poultry will eat less initially, and then increase their feed intake. (Siegel, 1995). During stress, nutrients in the feed are not digested and 
absorbed efficiently, and the animal must rely on the nutrient reserves of the body. These reserves are very important and help to sustain the animal during the stress. The muscle and liver carbohydrate stores (glycogen) are immediately called upon to furnish energy. Protein is broken down to yield the glucogenic and ketogenic amino acids which, following deamination, will supply the bird with energy. This energy from carbohydrate and protein allows the bird to maintain its health and survival. During stress the vital functions of the brain, liver, heart, lungs, kidney, etc. cannot be compromised. Therefore, the less important functions such as egg production, reproduction, growth and immunity are set aside to promote the vital functions of the body in stressful situations. Immediate survival has the number one priority in all animals when they are confronted with a severe stressor. The full genetic potential of the bird for growth and egg production is not expressed during stress.

The shift in metabolism during stress favors fat deposition. This is contrary to what is happening to protein and glycogen stores in the bird, but carcass data (muscle depletion and fat accretion) confirm that muscle protein declines and fat deposition increases (Nagra and Meyer, 1963; Bartov et al., 1980; Siegel and Van Kampen, 1984). During stress the consumption of water increases as a result of the necessity to clear the additional uric acid excretion arising from protein breakdown (Siegel and Van Kampen, 1984). The increased water consumption is also probably necessary to maintain osmolality in the body fluids due to the increased sodium retention concurrent with the effects of corticosterone (Holmes and Phillips, 1976).

\section{Adrenal Gland, Stress Hormone and Nutrient Requirements}

Corticosterone is the main stress hormone that is produced and released from the cortex of the adrenal gland when an animal is confronted with stress. This hormone influences the metabolism of the energy-yielding nutrients. Corticosterone is responsible for ensuring that the nutrient stores are allowed to furnish the energy the animal must have in order to cope with the stress. This hormone is responsible for increased use of glucose and amino acids for energy. A high rate of muscle protein synthesis does not occur in the presence of this hormone since the carbon skeletons of the amino acids are used for energy.

Most of the research in Universities and Research Centers conducted to determine the nutritional requirements is done under ideal situations for each species of animal, especially poultry. However, under commercial conditions, ideal environments do not always exist. Poultry are continually exposed to various stressors that often have a negative effect on their nutritional requirements. To the nutritionist, this "negative" effect is an increase in the dietary requirement for several nutrients.

Teeter and Wiernusz (1994) discussed how new management approaches could be used to allow poultry to cope and adapt to stressful situations. In their review these authors discussed how environmental effects, such as ambient temperature, could have a negative effect on performance and how the bird needed to adapt to high temperatures in order to survive. Growth rate was sacrificed in order that survival have the top priority. These authors also discussed how the bird was able to respond in growth following a stressful encounter. Bird growth rate during the "recovery phase" can possibly be accelerated by either a more rapid return to homeostatic conditions, or via a classical compensatory gain response. The daily gain in excess of that observed for non-stressed control animals following a period of stress is commonly known as compensatory gain (Teeter and Wiernusz, 1994). It has been shown that in most stressful situations, the breast muscle of the bird, especially the broiler, is the first muscle to lose protein and the last muscle to rebuild protein to replenish breast mass. The data presented by Teeter and Wiernusz (1994) indicated that the viscera, leg and thigh muscles all have a greater potential for compensatory gain than the breast muscle when a stress-free period follows a period of stress.

Other researchers have shown that the environmental temperature has an influence on the immune response of poultry (Beard and Michell, 1987; Donker et al., 1990; Henken et al., 1982). 
Siegel and Latimer (1984) speculated that the increased activity of the adrenal gland due to heat stress increased the level of serum corticosteroids which resulted in the decreased performance of poultry. Also, Arjona et al., 1990 reported that high environmental temperature stress before or soon after hatching would induce resistance to extreme environmental temperature fluctuations later in adult life. This adaptation to high temperatures brought about by early exposure has also been reported by Tetter and Wiernusz (1994). Thaxton and Siegel (1970) reported that the influence of environmental temperature variations on the immune response depends on the degree of adaptation of the animal and the time of immunization.

\section{Vitamin C}

As stated previously, the stress hormone corticosterone is responsible in all physiological stress for the channeling of nutrients and body reserves away from the economically important traits of poultry production to those vital physiological functions necessary for immediate bird survival. Therefore, any measure of reduction in the release of corticosterone allows nutrients to be used for growth, egg production immune response, etc. (Bains. 1996). Supplementation of vitamin C in stressful situations has been shown to be of benefit to the animal and has helped to restore some of the performance loss.

All poultry are capable of synthesizing vitamin $\mathrm{C}$ in the kidney tissue, and because of this, supplementation is often considered by a few nutritionists to be unnecessary. However, the synthesis and use of vitamin $\mathrm{C}$ are not constant. The ability of the kidney to synthesize vitamin $\mathrm{C}$ in the amount needed changes with age, management, environment, disease, nutrition and stress. In order to understand why vitamin $\mathrm{C}$ is beneficial in helping to alleviate stress it is necessary to consider vitamin $\mathrm{C}$ as an "anti-stress" vitamin and not a growth promotor in these situations. Some of the highest concentrations of vitamin $\mathrm{C}$ in animals can be found in the testicles, ovaries, and adrenal gland. In the adrenal gland, vitamin $\mathrm{C}$ functions metabolically to help control the production of the adrenal hormone, corticosterone. Stressors in the environment have a direct influence on plasma and tissue levels of vitamin C. A controlled rate of corticosterone release from the adrenal cortex is preferred in coping with stress. For adrenal cortical depletion of this hormone to occur would result in death of the animal. Vitamin $\mathrm{C}$ plays a central role in the continued synthesis of corticosterone. The proposed mechanism for this effect is through inhibition of the 21-hydroxylase and 11 beta-hydroxylase enzymes in the steroid biosynthetic pathway in the adrenal cortex. Vitamin C supplementation to the diet and water during periods of stress causes reduced synthesis of corticosterone (Brake, 1988). For best results, the use of vitamin $\mathrm{C}$ in the diet or water should begin at least 24 or 48 hours before the onset of stress and should continue throughout the stressful period. The recommended amount of vitamin $\mathrm{C}$ to use in the diet is usually between 100 and $150 \mathrm{ppm}$. Higher levels can be used, but are often cost prohibitive.

Vitamin $\mathrm{C}$ is not the only vitamin that should be supplemented during periods of stress. Research has shown that all vitamins should be increased in the diet if the stress level in poultry is high. Without these supplemental vitamins, acceptable performance and carcass characteristics will not occur. Vitamins also enhance disease resistance under conditions of stress. These vitamins exert their mechanism of action during stress by protecting immune tissue via a reduction of certain hormonal effects and oxidative damage, and enhancment of cell differentiation and production.

Stressed animals are less resistant to disease challenge and are at a greater risk from infection than non-stressed animals because of an impaired immune response. The glucocorticoids are known to be immnosuppressive (Golub and Gershwin, 1985). In stress, immune cell function is adversely affected by increased eicosanoid levels. Various stimuli, including stress hormones, activate phospholipase $\mathrm{A}_{2}$ which frees the 20-carbon fatty acid, arachidonic acid, from membrane phospholipids. As a by product of this breakdown of cellular membranes and the production of prostaglandins from arachidonic acid, reactive oxygen molecules $\left(\mathrm{H}_{2} \mathrm{O}_{2}, \mathrm{OH}\right.$ and $\left.\mathrm{O}_{2}\right)$ are formed and the free radicals can cause damage to cell membranes. These free radicals can also result in the disruption of protein function, DNA structure, and energy production within the cell (Freeman and 
Crapo, 1982). This is why the antioxidant compounds (vitamin $\mathrm{E}$ and beta carotene) are also beneficial during stress. As stated by Nockels et al., (1986), whatever reduces immunosuppressive prostaglandin production and limits cellular membrane levels of its precursor, arachidonic acid, should affect disease resistance.

Stress also causes the animal to increase its metabolic rate, which through normal oxidative metabolic pathways produces increased free radicals. Increased mineral losses have been reported in stressful conditions, which reduce metalloenzymes necessary for free radical and peroxide removal from the body (Nockels, 1990). This is another reason that the need for antioxidants in the diet will usually increase during stress. Vitamins $\mathrm{E}$ and $\mathrm{C}$ are very important during disease stress, such as in infection. In infection, phagocytes destroy ingested particles by generating free radicals which may prove self destructive if antioxidant systems are inadequate. Phagocytic function and surrounding tissues can be protected by feeding vitamins E and C (Boxer, 1986; Anderson and Lukey, 1987). Vitamin C helps to regenerate vitamin $\mathrm{E}$ by reducing vitamin $\mathrm{E}$ radicals formed when vitamin $\mathrm{E}$ scavenges oxygen radicals (Niki, 1987).

Disease resistance is also a function of cell differentiation and one of the primary functions of vitamin $\mathrm{A}$ is to maintain proper epithelial tissue differentiation and prevent epithelial keratinization which occurs in a deficiency (Chytil et al., 1983). Vitamin A is required for maintaining cellularity of lymphoid organs, which are essential in combating disease stress. Vitamin A is also required for enhancing both cellular and humoral immunity and enhances phagocyte activity (Chew, 1987). Vitamin A has reduced mortality in chicks infected with coccidial oocysts from E. tenella and E. acervulina (Erasmus et al., 1960). Since vitamins A, E, C and carotenoids are able to protect cells from free radical oxidation, reduce the detrimental effects of certain eicosanoids, and enhance humoral and cellular immune responses in disease stress, nutritionists must continually redefine the levels of vitamins necessary to maximize health during stress and disease (Nockels, 1989).

\section{Trace Mineral Requirements}

Stress decreases the circulating levels of trace minerals in animals, causes greater endogenous loss, decreases the efficiency of trace minerals and increases the metabolic need to fight the stress. Because of this, nutritionists often without question will increase the dietary concentration of trace minerals when poultry and other animals are confronted with a stressful situation. During stress, increased serum copper and decreased serum iron, zinc and manganese have been reported (Klasing et al., 1991). One of the most serious stressors that can confront poultry is disease. Trace mineral metabolism is radically changed during disease challenge because of the demand by a family of acute phase proteins that are central to the early phases of the immune response.

Zinc concentrations in the serum are decreased as a result from the redistribution of zinc from the plasma pool to a newly synthesized metallothionein pool in the liver and other tissues. Metallothionein induction supplies zinc to be used as a cofactor for metalloproteinases and other acute phase proteins (Klasing et al., 1991).

Copper-containing ceruloplasmin serves as a protective antioxidant and is a necessary component of the acute phase response. The quantity of copper required to sustain this role is large relative to other functions of copper. A copper deficiency reduces the amount of ceruloplasmin which is released during an inflammatory stress (DeSilvestro and Marten, 1990).

One of the most interesting mechanisms of defense that animals have to fight infection is to remove the circulating iron from circulation. During infection, iron is removed from the circulation and sequestered into compartments which are nutritionally unavailable to bacteria and parasites. Weinberg (1974) names this iron sequesting process "nutritional immunity." It has been shown that if the iron lost from circulation is replenished by injections or very high dietary supplementation, increases in mortality and morbidity will occur in a variety of diseases (Beisel, 1977). The absorption of iron from the digestive tract is markedly reduced within hours after a disease challenge. 
Manganese needs by the gastrointestinal tract, lungs and several other tissues in the bird are increased during an immune response. The reason for this is that manganese serves as a cofactor of superoxide dismutase and ameliorates damage induced by the immune response itself. However, there is very little change in the circulating manganese levels in the plasma during stress (Klasing, et al., 1991).

The goal of trace mineral supplementation during stress should be to provide a readily available form that can be absorbed and give replete storage pools prior to the time that stress is encountered. These storage pools of the trace minerals will buffer the low levels due to stress and facilitate the greater tissue accretion that follows an encounter with stress (Klasing, et al., 1991).

\section{Summary}

Stressors are a part of every poultry operation. Management of these stressors to minimize the stress response in the bird should be the goal of every good poultry manager. Stress will not allow the bird to express its full genetic potential for growth, feed conversion and egg production. Stress also depresses the bird's ability to mount a successful immune response. Modern poultry are continuously and simultaneously exposed to a number of stressors. The successful poultry enterprise is the one in which the nutritionist, production manager, veterinarian and other personnel have an understanding of stress and make an attempt to do everything possible to minimize the stressors in the operation. The three way interrelationship which exists among stress, nutrition and immunity must also be kept in mind. Using common sense to solve the everyday problems encountered in poultry operations will go a long way in helping poultry flocks to cope with the stressors in their environment. Stress is unavoidable, but it can be managed.

\section{References}

Arjona, A. A., D. M. Denbow, and W. D. Weaver, Jr., 1990. Neonatally induced thermotolerance. Comp. Bioch. Physiol. 95 A:393-399.
Anderson, R. and P. T. Lukey, 1987. A biological role for ascorbate in the selective neutralization of extracellular phagocyte-derived oxidants. N. Y. Acad. Sci. 498:229-247.

Bains, B. S., 1996. The role of vitamin C in stress management. World Poultry-Misset, Vol. 12, no. 4 , pp. 38-41.

Bartov, I., L. S. Jensen and J. R. Veltmann, 1980. Effect of corticosterone and prolactin on fattening in broiler chicks. Poultry Sci. 59:1328-1334.

Beard, C. W. and B. W. Michell, 1987. Influence of environmental temperatures on the serological responses of broiler chickens to inactivated and viable Newcastle disease vaccines. Avain Dis. 31:321-326.

Beisel, W. R., 1977. Magnitude of the host nutritional responses in infection. Am. J. Clin. Nutr. 30:1236-1247.

Benedich, A., ed., 1990. Antioxidant vitamins and their function in immune response. Pages 35-55 in: Advances in Experimental Medicine and Biology, Vol. 262. Plenum Press, New York, NY.

Berczi, I., D. A. Chow and E.R. Sabbadini, 1998. Neuroimmunoregulation and Natural Immunity. Domestic Animal Endocrinology, Vol. 15(5):273-281.

Blumberg, J., 1994. Vitamins. Pages 234-247 in: Diet, Nutrition, and Immunity. R. A. Forse, ed. CRC Press, Baca Raton, FL.

Boxer, L. A., 1986. Regulation of phagocyte function by alpha-tocopherol. Proc. Nutr. Soc. 45:333-334.

Brake, J. T., 1988. Stress of birds, modern poultry management relationship defined. Poultry Digest, May, pp. 226-231.

Chew, B. P., 1987. Symposium: Immunofunction: Relationship of nutrition and disease control. Vitamin A and beta-carotene on host defense. J. Dairy Sci. 70:2732-2743. 
Chytil, R., M. Omori, G. Liau and D. E. Ong, 1983. Function of vitamin A in $\mathrm{C}$ differentiation. Fed. Proc. 42:2744-2753.

Collier, J. and P. Vallance, 1989. Second messenger role for $\mathrm{NO}$ widens to nervous and immune systems. Trends Pharmacol. Sci. 10:427-431.

Curtis, S. E. 1983. Environmental aspects of housing for animal production. Iowa State Univ. Press, Ames, IA

DeSilvestro, R. A., J. T. Marten, 1990. Effects of inflammation and copper intake on rat liver and erythrocyte $\mathrm{Cu}-\mathrm{Zn}$ superoxide dismutase activity levels. J. Nutr. 120:1223-1227.

Donker, R. A., M. G. B. Nieuwland, and A. J. Van Der Zijpp, 1990. Heat-stress influences on antibody production in chicken lines selected for high and low immune responsiveness. Poult. Sci. 69:599-607.

Efron, D. T. and A. Barbul, 1998. Modulation of inflammation and immunity by arginine supplements. Curr. Opin. Clin. Nutr. Metab. Care 1:531-538.

Erasmus, J., M. L. Scott and P. P. Levine, 1960. A relationship between coccidiosis and vitamin A nutrition in chickens. Poultry Sci. 39:565-571.

Evoy, D., M. D. Lieberman, T. J. Fahey, III, and J. M Daly, 1998. Immunonutrition: The role of arginine. Nutrition 14:611-617.

Freeman, B. A. and J. D. Crapo, 1982. Biology of disease free radicals and tissue injury. Lab. Invest. 47:412-426.

Friend, T. H. and G. R. Dellmeier, 1986. Recent developments in stress research. Prof. Anim. Sci., pp. $1-6$.

Gershwin, M., R. Beach and L. Hurley, 1985. The potent impact of nutritional factors on immune response. Pages 1-7 in: Nutrition and Immunity. Academic Press, New York, NY.

Golub, M. S. and M. E. Gershwin, 1985. Stress-induced immunomodulation: What is it, if it is? In: Animal Stress. Gary P. Moberg (ed.) Am. J. Physiol. Soc., Bethesda, MD, pp. 177-192.
Harvey, S., J. G. Phillips, A. Rees, and T. R. Hall, 1984. Stress and adrenal function. J. Exp. Zoo, 232:633-645

Henken, A., A. M. J. Groote Schaarsberg and M. G. B. Nieuwland, 1982. The effect of environmental temperature on immune response and metabolism of the young chicken. 3. Effect of environmental temperature on the humoral immune response following injection of sheep red blood cells. Poult. Sci. 62:51-58.

Holmes, W. N. and J. G. Phillips, 1976. The adrenal cortex of birds, in: I. Chester-Jones \& I. W. Hnderson (Es) General, Comparative and Clinical Endocrinology of the Adrenal Cortex, Vol. 1, pp. 292-420. (New York, Academic Press).

Kidd, M. T., E. D. Peebles, S. K. Whitmarsh, J. B. Yeatman and R. F. Wideman, Jr., Growth and immunity of broiler chicks as affected by dietary arginine. Poultry Sic. 80:1535-1542.

Klasing, D. C., B. J. Johnstone, and B. N. Benson, 1991. Implications of an immune response on growth and nutrient requirements of chicks. In: Recent Advances in Animal Nutrition (Haresign, W., Cole, D. J. A., ed), Butterworth Heinemann.

Klasing, 1997. Interaction between nutrition and infectious disease. Pages 73-80 in: Diseases of Poultry, B. W. Calnek, ed. Iowa State University Press, Ames, IA.

Klasing, K. C., 1998. Avian macrophages: Regulators of local and systemic immune responses. Poultry Sci. 77:983-989.

Leshchinsky, T. V. and K. C. Klasing, 2001. Relationship between the levels of dietary vitamin E and the immune response of broiler chickens. Poultry Sci. 80:1590-1599.

Nagra, C. L. and Meyer, R. K., 1963. Influence of corticosterone in the metabolism of palmitate and glucose in cockerels. Gen. And Comp. Endo., 3:131-138.

National Research Council, 1994. Nutrient Requirement of Poultry. $9^{\text {th }}$ Rev. Ed. National Academy Press, Washington, DC. 
Niki, E., 1987. Interaction of ascorbate and alpha-tocopherol. N. Y. Acad. Sci. 498:186-198.

Nockels, C. F., 1986. Dietary fatty acids and vitamin E alter immune organ lipids. Fed. Proc. 45:353-360.

Nockels, C. F., 1989. Vitamin needs increase during stress, disease. Poultry Digest, May, pp. 218-226.

Nockels, C. F., 1990. Mineral alterations with changing environment. In: Proceedings $51^{\text {st }}$ Annual Conference for Veterinarians. January 6-9, Colorado State University, Ft. Collins, CO, pp. 461-474.

Packer, L. and Y. Suzuki, 1993. Vitamin E and alpha-lipoate: Role in antioxidant recycling and activation of the NK-B transcription factor. Mol. Asp. Med. 14:229-239.

Selye, H., 1973. The evolution of stress concept. American Scientist, Vol. 61: Nov.-Dec., pp. 692-699.

Siegel, H. S. and M. Van Kampen, 1984. Energy relationships in growing chickens given daily injections of corticosterone. British Poult. Sci., 25:471-485.

Siegel, H. S. and J. W. Latimer, 1984.

Interaction of high temperature and Salmonella pullorum antigen concentration on serum agglutinin and corticosteroid responses in white rock chickens. Poultry Sci. 63:2483-2491.

Siegel, H. S., 1995. Stress, strains and resistance. British Poult. Sci., 36:003-22. 